\title{
Sensibilidad Paramétrica y Condiciones Seguras de Operación de la Hidrólisis del Anhídrido Acético en un Reactor Batch
}

\author{
Guillermo H. Gaviria(1a), Edison GilPavas ${ }^{(1,2)}$, Izabela Dobrosz-Gómez ${ }^{(1 b, 2)}$ y Miguel Á. Gómez ${ }^{(1 a, 2)}$ \\ (1) PRISMA: Grupo de Investigación en Procesos Reactivos Intensificados con Separación y Materiales \\ Avanzados. aDepartamento de Ingeniería Química, Facultad de Ingeniería y Arquitectura. bDepartamento de \\ Física y Química, Facultad de Ciencias Exactas y Naturales. Universidad Nacional de Colombia, Sede \\ Manizales, Campus La Nubia, Apartado Aéreo 127, Manizales, Caldas, Colombia \\ (e-mail: ghgavirial@unal.edu.co; idobrosz-gomez@unal.edu.co; magomez@unal.edu.co) \\ (2) GIPAB: Grupo de Investigación en Procesos Ambientales, Universidad EAFIT, Medellín, Colombia \\ (e-mail: egil@eafit.edu.co)
}

Recibido Oct. 28, 2015; Aceptado Dic. 29, 2015; Versión final Feb. 9, 2016, Publicado Ago. 2016

\begin{abstract}
Resumen
Se presentan los resultados de un estudio de sensibilidad paramétrica para el proceso de producción de ácido acético mediante hidrólisis de anhídrido acético bajo condiciones de catálisis ácida, en un reactor batch. Se ajustaron parámetros de los balances adimensionales de materia y energía a partir de datos experimentales disponibles en la literatura, los cuales incluyen la variación de la temperatura de reacción para diferentes concentraciones de ácido sulfúrico (3\% y 5\%). Luego, a partir del análisis de las propiedades matemáticas intrínsecas del plano de fases concentración-temperatura, se definen las condiciones críticas del proceso. Así, se logra determinar valores puntuales de condiciones seguras de operación para la hidrólisis catalizada de anhídrido acético, en función de la concentración de catalizador.
\end{abstract}

\section{Parametric Sensitivity and Safe Operating Conditions of Acetic Anhydride Hydrolysis in a Batch Reactor}

\begin{abstract}
The results of a study on the parametric sensitivity of acetic acid production by hydrolysis of acetic anhydride, using acid homogeneous catalysis in a batch reactor, are presented. Dimensionless parameters of mass and energy balances were fitted using experimental data available in the literature that includes the variation of the reaction temperature for different sulfuric acid concentrations (3\% to $5 \%$ ). Then, from the analysis of the intrinsic mathematical properties of the concentration-temperature phase plane, the critical operating conditions were defined. Thus, it was possible to determine the specific values of the safe operating conditions for the catalyzed hydrolysis of acetic anhydride, in the function of catalyst concentration.
\end{abstract}

Keywords: parametric sensitivity; acetic anhydride; acid catalysis; acetic acid; batch reactor. 


\section{INTRODUCCIÓN}

El ácido acético, ácido metilcarboxílico o ácido etanoico, es un ácido que se encuentra en el vinagre, siendo el principal responsable de su sabor agrio y olor acre. Es de interés para la química orgánica como reactivo, para la química inorgánica como ligando, y para la bioquímica como metabolito. Este se obtiene principalmente mediante procesos de síntesis (v.g., carbonilación del metanol, oxidación del acetaldehído o del etileno, hidrólisis del anhídrido acético, entre otros) o por fermentación bacteriana (oxidativa: Acetobacter, anaerobia: Clostridium) (Kirk-Othmer, 2007).En cuanto a su uso, tiene dos áreas principales de aplicación comercial: i) vinagre de calidad alimentaria, que se obtiene por oxidación bacteriana de alcohol diluido procedentes de diversas frutas y granos, ii)ácido acético industrial sintetizado químicamente, que se usa industrialmente para la síntesis de vinil acetato, ácido cloroacético, éteres de acetatos, entre otros (Rogerset al.2006). Transparency Market Research (2015) reportó que el mercado mundial de ácido acético fue valorado en USD 5,93 millones en 2011 y se espera que llegue a USD 10.31 millones de dólares en 2018. En términos de volumen, la demanda mundial era 9,612.5 kilo toneladas en 2011 y se espera que crezca a una tasa compuesta anual del 4,8\% durante el período pronosticado 2012-2018.

Haldar y Rao (1992) estudiaron en un reactor por lotes (o batch en inglés) la hidrólisis del anhídrido acético en fase líquida, catalizada homogéneamente por ácido sulfúrico, para obtener ácido acético. Sus resultados demostraron que esta reacción, moderadamente exotérmica $\left(\Delta H_{r x n}=-58,52 \mathrm{~kJ} / \mathrm{mol}\right.$, obtenido con los parámetros tabulados en Poling et al., 2000), es muy sensible a la variación de las condiciones de operación. De hecho, un cambio de 0,5 K en la temperatura de alimento, de 319 a 319,5 K, generó un cambio en la temperatura en el sistema de aproximadamente $31 \mathrm{~K}$. Más recientemente, Jayakumar et al. $(2011,2014)$ estudiaron este proceso reactivo en un reactor de mezcla completa (o CSTR de su sigla en inglés) y concluyeron que el sistema en estado dinámico tiene comportamiento oscilatorio y a ciertas condiciones de operación exhibe sensibilidad paramétrica. Gómez et al. (2015) realizaron un estudio sobre la estabilidad térmica y el comportamiento dinámico de la reacción en mención en el mismo tipo de reactor y demostraron que el sistema es sensible a pequeñas perturbaciones en el fluido de refrigeración. Por consiguiente, se puede decir que el proceso de hidrólisis del anhídrido acético exhibe sensibilidad paramétrica, tanto a las condiciones de operación como al modo de operación. Para el análisis de estos fenómenos reactivos se han propuesto y desarrollado herramientas matemáticas eficaces (Adroveret al., 2007). Uno de los objetivos de mayor interés cuando se hace este tipo de estudios es determinar las condiciones de operación que conllevan a la manifestación de fugas térmicas (del inglés thermalrunaway). Estas se caracterizan por el aumento progresivo de la velocidad de generación de calor, temperatura, y presión. Las fugas térmicas pueden ocasionar dificultades en el desempeño del proceso reactivo: problemas de selectividad y rendimiento (reacciones laterales), daño permanente en equipos y catalizadores, aumentos de temperatura y presión, que hacen que el sistema pueda exceder los límites de operación segura. Así, durante las últimas dos décadas, se ha generalizado el uso de diferentes metodologías basadas en diferentes propiedades de los sistemas reactivos. Cassonet al. (2012) clasificaron los criterios en dos categorías: basados en la geometría (Semenov, Bowes y Thomas, Adler y Enig, van Welsenaere y Froment, Barkelew); basados en sensibilidad (Hub y Jones, Strozzi y Zaldivar). Por otro lado, los criterios para establecer condiciones seguras de operación se pueden clasificar en tres categorías: el método de sensibilidad paramétrica (Morbidelli y Varma, 1988; Varmaet al., 1999); el método de la divergencia basado en la teoría del caos (Strozzi, y Zaldivar, 1994) y el método de la extensión de trayectorias (Bosch et al., 2004). Además, Westerterp y Molga (2004) propusieron una metodología basado en diagramas de operación segura para reactores batch y semibatch. Este tipo de diagramas posteriormente fueron implementados por Maestri y Rota (2005) y por Milewska y Molga (2010) quienes incorporaron modelos de dispersión usando CFD.

En el presente trabajo, se extiende la metodología de análisis geométrico de Froment et al. (2011) para estudiar el comportamiento de la hidrólisis de anhídrido acético utilizando diferentes concentraciones de catalizador. Para este sistema reactivo, se encuentran disponibles en la literatura datos experimentales de la variación de la temperatura de reacción en función de la concentración del ácido sulfúrico (Shukla y Pushpavanam, 1994).Así, con base en esos resultados experimentales, se construye el plano de fases temperatura - concentración a partir del cual se deducen dos criterios concretos para el sistema bajo estudio. Los resultados obtenidos se comparan con los reportados por Shukla y Pushpavanam (1994), quienes realizaron un análisis de sensibilidad paramétrica básico fundamentado en los criterios propuestos por Barkelew (1959) y Villermaux y Georgakis (1991).

\section{SISTEMA REACTIVO}

La hidrólisis del anhídrido acético en presencia de ácido sulfúrico como catalizador homogéneo se puede presentar según la siguiente estequiometría: 


$$
\left(\mathrm{CH}_{3} \mathrm{CO}\right)_{2} \mathrm{O}+\mathrm{H}_{2} \mathrm{O} \stackrel{\mathrm{H}_{2} \mathrm{SO}_{4}}{\longrightarrow} 2 \mathrm{CH}_{3} \mathrm{COOH}
$$

El mecanismo de la hidrólisis de anhídrido acético ha sido ampliamente estudiado. La reacción procede a través de una sustitución, en la que un nucleófilo reemplaza un grupo sustituyente sobre el carbono central (Aspreyet al., 1996).La reacción es de pseudo-primer orden con respecto al anhídrido acético. La cinética para la hidrólisis catalizada por ácido fue reportada por Haldar y Rao (1991):

$$
r=k_{0} \cdot \exp \left(-\frac{E_{a}}{R T}\right) C_{S} C_{A}
$$

Donde $C_{A}$ y $C_{S}$ son las concentraciones de anhídrido acético y catalizador, respectivamente, en $\mathrm{mol} / \mathrm{L}$, T es la temperatura del sistema, en $\mathrm{K}, k_{0}$ es el factor de frecuencia $=1.85 \times 10^{14}$ mol.L.s $^{-1}, y E_{a} / R=9750 \mathrm{~K}$ (Haldar y Rao, 1991).

\section{MODELO MATEMÁTICO}

Los balances de materia y energía para un reactor por lotes con mezcla homogénea, con temperatura de pared constante, una única reacción irreversible de primer orden y densidad de fluido constante, pueden escribirse como (Froment et al., 2011):

$$
\begin{aligned}
& \frac{d_{C_{A}}}{d t}=-k_{0} C_{s} C_{A} \exp \left(-\frac{E_{a}}{R T}\right) \\
& \frac{d T}{d t}=\frac{\left(-\Delta H_{r \times n}\right)}{\rho C_{p}}\left(\frac{-d C_{A}}{d t}\right)-\frac{U A}{m C_{p}}\left(T-T_{w}\right)
\end{aligned}
$$

Donde $\Delta H_{r x n}$ es el calor de reacción, $\rho$ es la densidad de la mezcla, $C_{p}$ es la capacidad calorífica, $U$ es el coeficiente global de trasferencia de calor, $A$ es el área para intercambio de calor, $m$ es la masa de reactivo y $T_{w}$ es la temperatura de pared.

Estas ecuaciones pueden reescribirse en términos adimensionales para facilitar su manipulación:

$$
\begin{aligned}
& \frac{d C}{d t}=-A \cdot C_{A} \exp \left(-\frac{a}{T}\right) \\
& \frac{d T}{d t}=B \cdot C_{A} \exp \left(-\frac{a}{T}\right)-\beta\left(T-T_{w}\right)
\end{aligned}
$$

Donde

$$
\mathrm{A}=\mathrm{k}_{0} \mathrm{C}_{\mathrm{s}} \quad \mathrm{B}=\frac{\left(-\Delta \mathrm{H}_{\mathrm{rxn}}\right) \mathrm{k}_{0}}{\rho \mathrm{C}_{\mathrm{p}}} \mathrm{C}_{\mathrm{s}} \quad \beta=\frac{\mathrm{UA}}{\mathrm{mC}_{\mathrm{p}}} \quad \mathrm{a}=\frac{\mathrm{E}_{\mathrm{a}}}{\mathrm{R}}
$$

Shukla y Puspavanam (1994) reportaron que, para condiciones normales de operación, el parámetro $\beta$ tiene un valor de $0.001 \mathrm{~s}^{-1}$, mientras la temperatura inicial del proceso reactivo sea de $294,15 \mathrm{~K}$, y la temperatura de pared del reactor oscile entre 293,15 y 297,15 K. Además, Shukla y Puspavanam (1994) reportaron un valor para el factor de frecuencia correspondiente a $1,85 \times 10^{14} \mathrm{~L} \cdot \mathrm{mol}^{-1} \cdot \mathrm{s}^{-1}$ y un valor de $\mathrm{E}_{\mathrm{a}} / \mathrm{R}$ de $11224 \mathrm{~K}$.

\section{Comportamiento del reactor}

Froment et al. (2011) propusieron dos criterios para el análisis de sensibilidad paramétrica de un sistema reactivo. Estos están basados en las propiedades matemáticas intrínsecas del modelo simplificado para el reactor tubular con una única reacción y sirven, entre otras cosas, para establecer las condiciones críticas de alimento al reactor. En este trabajo, considerando la analogía matemática que existe entre el modelo ideal del reactor tubular y el modelo de un reactor por lotes, se extiende dicha metodología de análisis con la construcción del plano de fases concentración-temperatura para un reactor por lotes. Así, inicialmente, se divide la ecuación (6) entre la ecuación (5) para obtener: 


$$
\frac{d T}{d_{A}}=-\frac{B}{A}+\frac{\beta}{A} \frac{\left(T-T_{w}\right)}{C_{A} \cdot \exp (-a / T)}
$$

Con la ecuación (7) se obtienen las trayectorias en el diagrama $C$ - $T$ mediante integración numérica usando MatLab $^{\circledR}$ (www.matworks.com). En estas trayectorias son de especial interés la localización del lugar geométrico (locus) de los máximos y de los puntos de inflexión en los perfiles de temperatura del reactor (Froment et al., 2011).

Se busca establecer una función que describa los máximos de temperatura en el reactor. Para cada concentración de anhídrido existe una temperatura máxima o punto caliente. Matemáticamente, esto ocurre cuando la derivada de la temperatura respecto al tiempo es igual a cero, o dicho de otra forma, igualado a cero la ecuación (6):

$$
B \cdot C_{A, m} \cdot \exp (-a / T)=\beta\left(T_{m}-T_{w}\right)
$$

Donde $C_{A, m}$ es la concentración de anhídrido en el punto de temperatura máxima, $T_{m}$. Esta concentración puede establecerse de forma explícita:

$$
C_{A, m}=\frac{\left(T_{m}-T_{w}\right)}{\frac{B}{\beta} \exp (-a / T)}
$$

La ecuación (9) solo tiene como variable la temperatura de pared. Esta curva representa la ubicación de los puntos calientes para diferentes temperaturas de pared. Adicionalmente, si se deriva la ecuación (9) con respecto a $T_{m}$ y se iguala a cero, se encuentran los máximos de la curva de puntos calientes $\left(T_{M}\right)$ :

$$
\mathrm{T}_{\mathrm{M}}=\frac{1}{2}\left[\mathrm{a}-\sqrt{\mathrm{a}\left(\mathrm{a}-4 \mathrm{~T}_{\mathrm{w}}\right)}\right]
$$

Cuando se incorporan la ecuación (9) al plano de fases $C$-T, esta pasa por todos los puntos calientes del sistema. A su vez, la ecuación (10) permite identificar el máximo de la curva de puntos calientes. Después de este punto, pequeños incrementos en la concentración del alimento generan aumentos drásticos en la temperatura del sistema. Este será el Criterio 1de análisis, tal como lo propusieron originalmente Froment et al. (2011). Esta condición corresponde a la concentración inicial de la trayectoria en el plano $C$ - $T$ que pasa por el punto máximo de la curva de puntos calientes.

El Criterio 2se basa en la localización de los puntos de inflexión en los perfiles de temperatura del reactor (a partir de los cuales el crecimiento de la función aumenta considerablemente). Estos puntos de inflexión de coordenadas $C_{A, i}$ y $T_{i}$ en el plano de fases $C$ - $T$, se presentan antes y después del máximo y se puede obtener derivando la ecuación (6) e igualándola a cero:

$$
\frac{\mathrm{a}}{\mathrm{T}_{\mathrm{i}}^{2}}\left[\frac{\mathrm{B} \cdot \exp \left(-\frac{\mathrm{a}}{\mathrm{T}_{\mathrm{i}}}\right)}{\beta} \mathrm{C}_{\mathrm{A}, \mathrm{i}}\right]^{2}-\left[1+\frac{\mathrm{a}}{\mathrm{T}_{\mathrm{i}}^{2}}\left(\mathrm{~T}_{\mathrm{i}}-\mathrm{T}_{\mathrm{w}}\right)+\frac{\mathrm{A}}{\beta} \exp \left(-\frac{\mathrm{a}}{\mathrm{T}_{\mathrm{i}}}\right)\right]\left[\frac{\mathrm{B} \cdot \exp \left(-\frac{\mathrm{a}}{\mathrm{T}_{\mathrm{i}}}\right)}{\beta} \mathrm{C}_{\mathrm{A}, \mathrm{i}}\right]+\left(\mathrm{T}_{\mathrm{i}}-\mathrm{T}_{\mathrm{w}}\right)=0
$$

La ecuación (11) expresa la relación Temperatura-Concentración en los puntos de inflexión en el plano Temperatura-tiempo. Para facilitar el uso de esta ecuación se hacen las siguientes agrupaciones algébricas:

$$
\begin{aligned}
& C^{*}=\frac{B \cdot \exp (-a / T)}{\beta} C_{A, i} \\
& A^{*}\left(T_{i}\right)=\frac{a}{T_{i}^{2}}
\end{aligned}
$$


$B^{*}\left(T_{i}\right)=1+\frac{a}{T_{i}^{2}}\left(T_{i}-T_{w}\right)+\frac{A}{\beta} \exp \left(-a / T_{i}\right)$

$\beta^{*}\left(T_{i}\right)=T_{i}-T_{w}$

Se reescribe la ecuación (11) en términos de las nuevas variables definidas por las ecuaciones (12)-(15).

$A^{*}\left(C^{*}\right)^{2}-B^{*} C^{*}+\beta^{*}=0$

Así, la ecuación (16), que tiene la forma de una expresión cuadrática, se puede resolver hallando las dos raíces que satisfacen esa igualdad:

$C_{1,2}^{*}=\frac{B^{*} \pm \sqrt{\left(B^{*}\right)^{2}-4 A^{*} \beta^{*}}}{2 A^{*}}$

Así que,

$$
\left(\mathrm{C}_{\mathrm{A}, \mathrm{i}}\right)_{1,2}=\frac{\mathrm{C}_{1,2}^{*}}{\frac{\mathrm{B}}{\beta} \exp \left(-\mathrm{a} / \mathrm{T}_{\mathrm{i}}\right)}
$$

Resulta evidente la presencia de dos valores de $C_{A, i}$ para cada valor de $T$. Como estos valores son aquellos en los que ocurre la inflexión de la curva de temperatura y están antes y después del máximo o punto caliente, solo se necesita evaluar la inflexión antes del máximo dado que esta es la curva que describe la condición crítica del sistema. Siendo así, la trayectoria crítica será aquella que sea tangente a la curva $\left(C_{A, i}\right)_{1}$ y por ende, la concentración de anhídrido y temperatura a las condiciones de entrada para una temperatura de pared fija serán consideradas críticas.

\section{RESULTADOS Y DISCUSIÓN}

En la Fig. 1 se presentan los perfiles experimentales de temperatura en función del tiempo para dos concentraciones diferentes de catalizador (Shukla y Puspavanam, 1994).

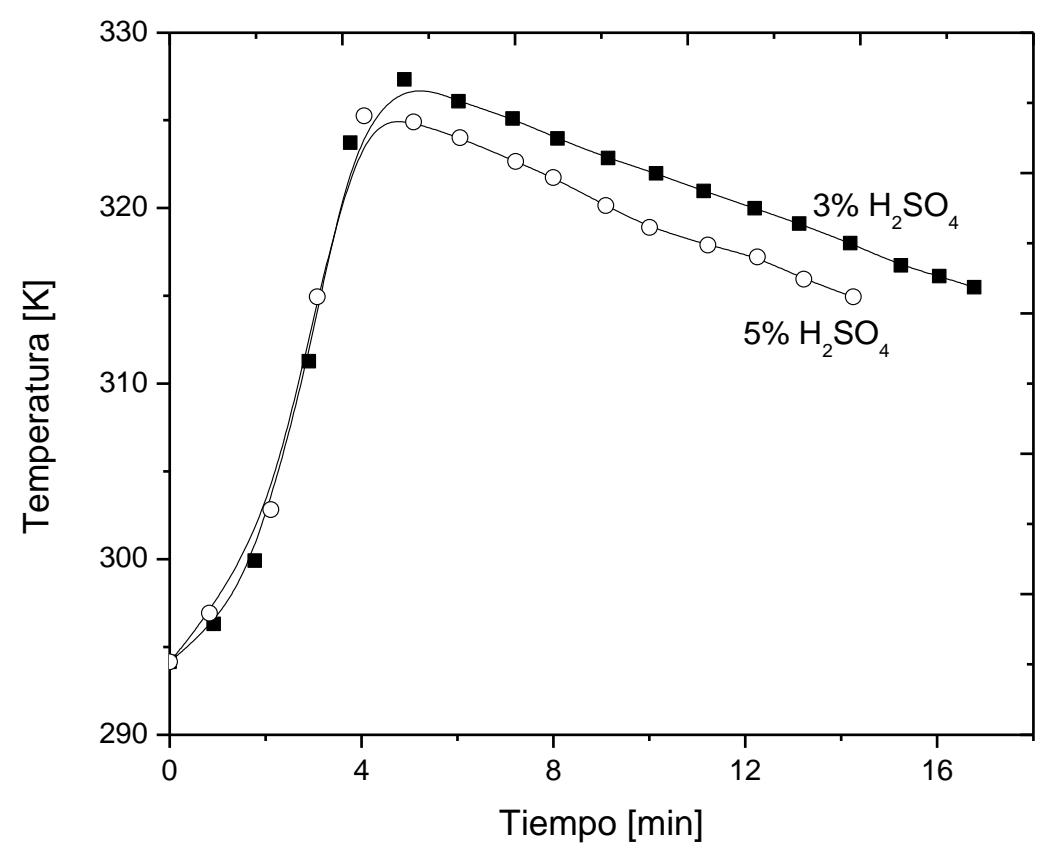

Fig. 1: Perfiles de temperatura en función del tiempo a diferentes concentraciones de $\mathrm{H}_{2} \mathrm{SO}_{4}$. Símbolos: son datos experimentales (Shukla y Pushpavanam, 1994); líneas son los resultados predichos por el modelo (se fijó como criterio de convergencia $0.001 \%$ ). 
En estos perfiles se puede apreciar la sensibilidad de la temperatura del sistema. En efecto, bajo condiciones de catálisis ácida, la temperatura se eleva hasta $40 \mathrm{~K}$ en los primeros 5 minutos de operación. Estos incrementos de temperatura pueden generar condiciones de operación no seguras. Como alternativa para evitar la elevación de temperatura se podría operar en modo semibatch, alimentando gradualmente los reactivos, sin embargo, Westerterp et al. (2014) demostraron que en este modo de operación el sistema también exhibe sensibilidad para métrica. La Fig.1 también incluye los datos obtenidos con el modelo propuesto. En este último, el parámetro de transferencia de calor $(B)$ fue ajustado en función de la concentración de catalizador obteniéndose un valor de $B=16.4 C_{s}$. La Fig. 1 muestra la bondad del ajuste. Aquí se observa que el aumento de temperatura para la reacción que incluye $3 \%$ de catalizador es superior al de $5 \%$. Esto ocurre porque al aumentar la cantidad de ácido sulfúrico, se incrementa también la capacidad calorífica de la mezcla reactiva.

Con el modelo matemático ajustado, se determinó el plano de fases $C$-Tpara la hidrólisis del anhídrido acético. Los resultados se muestran en la Fig. 2 para una concentración de catalizador $C_{s}=0.075 \mathrm{M}$, equivalente al $1 \%$ de ácido sulfúrico. Se escoge esta concentración de catalizador para mejorar la visualización del plano de fases $C$ - $T$. En ella se presentan además los resultados obtenidos para los dos criterios de sensibilidad. Así, es posible decir que las concentraciones críticas de anhídrido acético son de $1.46 \mathrm{M}$ y $1.69 \mathrm{M}$, para el Criterio 1 y el Criterio 2, respectivamente.

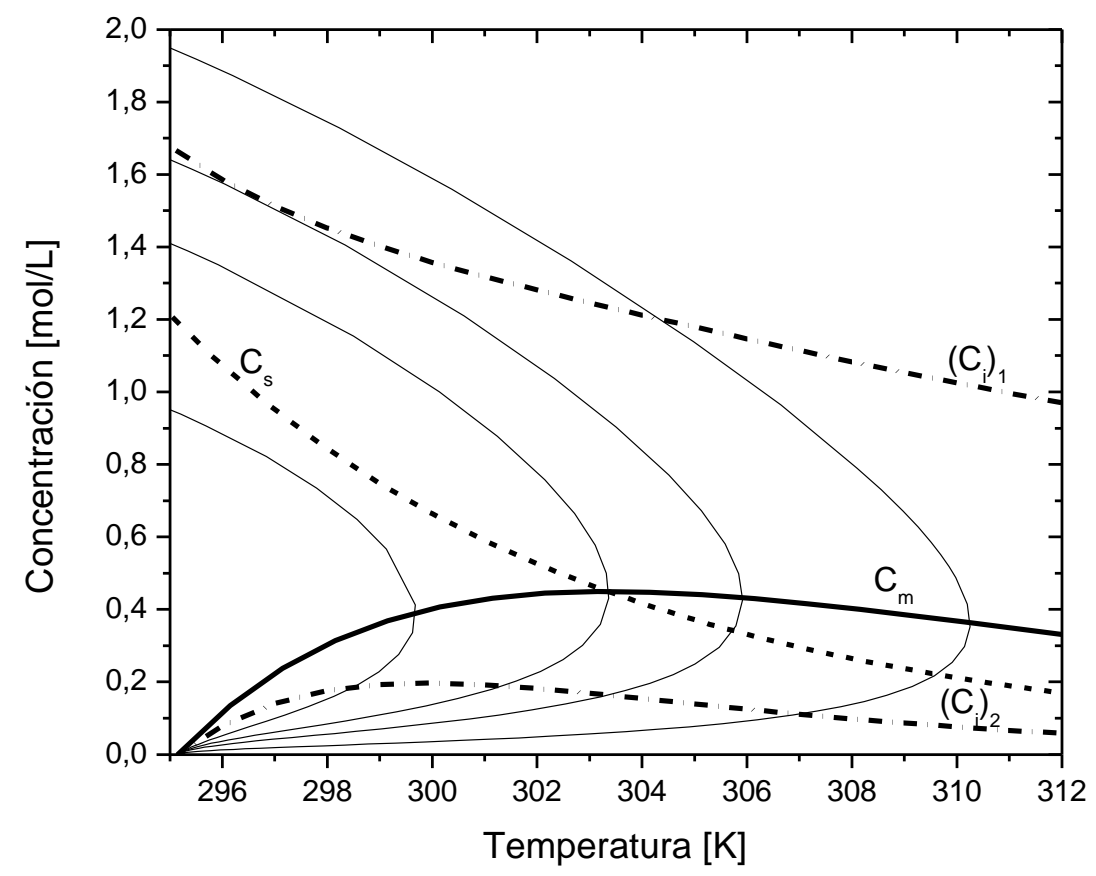

Fig. 2: Plano de fases $C$ - $T$ para la hidrólisis del anhídrido acético (1\% de $\mathrm{H}_{2} \mathrm{SO}_{4}$ ).Se detallan las trayectorias críticas para los dos criterios analizados.

De manera análoga, se determinaron las trayectorias críticas y las condiciones críticas de alimentación al reactor, usando los dos criterios, variando la concentración de catalizador. Estos resultados se presentan en la Fig. 3. Inicialmente, los valores de concentración de anhídrido acético calculados mediante el Criterio 1, son más conservadores (bajos) respecto a los valores obtenidos mediante el Criterio 2. Esta tendencia cambia para las cargas de catalizador mayores de $2.8 \%$ debido al cambio de pendiente de los perfiles de temperatura - tiempo dentro del reactor. A mayor carga de catalizador, mayor será la pendiente del perfil de temperatura al inicio de la reacción, y por ende, la inflexión de esta curva se presentará más rápidamente. Este comportamiento hace que, para cargas bajas de catalizador, la trayectoria que cruza por el máximo de los puntos calientes esté antes de la trayectoria tangente a la curva de puntos de inflexión de temperatura.

Finalmente, en la Fig. 4 se presentan los perfiles de temperatura y conversión respecto al tiempo, para las concentraciones críticas de anhídrido acético a 1 y $5 \%$ de catalizador. Se escogen estas dos concentraciones debido a que se encuentran a ambos lados del punto de corte de los dos criterios en la Fig. 3. Debido a que el aumento de concentración de catalizador conlleva a un descenso en la concentración crítica de anhídrido acético, los incrementos de temperatura son menores para altas concentraciones de ácido sulfúrico (Fig. 4a). 


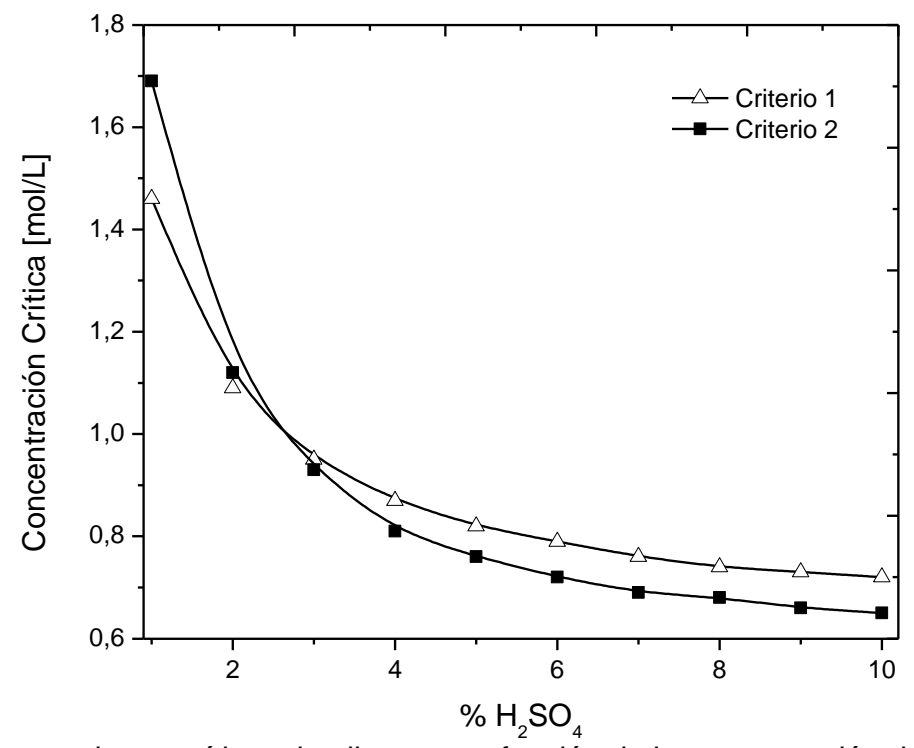

Fig. 3: Concentraciones críticas de alimento en función de la concentración de catalizador.

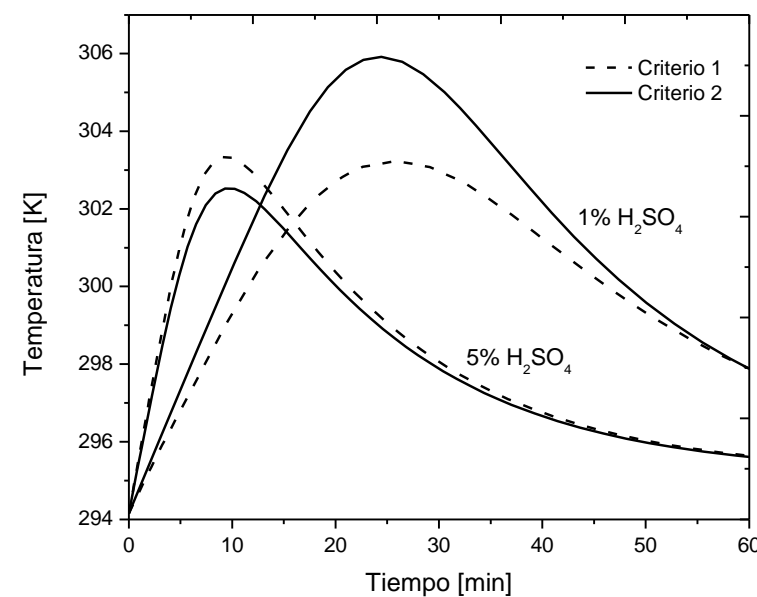

a)

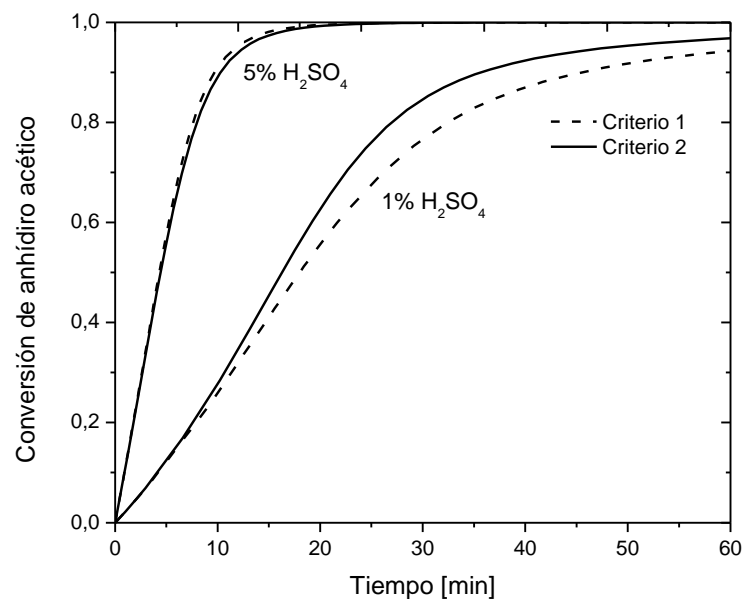

b)

Fig. 4:Perfiles de temperatura (a) y conversión (b) en función del tiempo para las concentraciones críticas de anhídrido acético a 1 y $5 \%$ de $\mathrm{H}_{2} \mathrm{SO}_{4}$

Analizando comparativamente los valores calculados por los dos criterios se observa que, para $1 \%$ de catalizador, los perfiles de temperatura de las concentraciones críticas halladas por ambos criterios, son muy similares y solo difieren en $2,68 \mathrm{~K}$ en su punto máximo de temperatura. Por otro lado, para concentración de $5 \%$ de catalizador, esta diferencia es de solo 0,81K. Cuando se incrementa la concentración de catalizador, la concentración crítica de anhídrido acético disminuye y por ende, la cantidad total de calor generado será inferior; esto hace que la temperatura de la mezcla reactiva no se eleve en gran magnitud. Al analizar la conversión del anhídrido acético se encuentra que, al aumentar la concentración de catalizador se llega a conversión completa más rápidamente sin importar el criterio usado para definir la concentración inicial de anhídrido (Fig. 4b). Sin embargo, es deseable obtener una alta conversión de reactivo, invirtiendo la menor cantidad de recursos posibles (cantidad de catalizador, tiempo de operación, fluido de servicio). En consecuencia, usando las concentraciones críticas de alimento de anhídrido acético en función de la concentración de catalizador, se puede plantear un problema de optimización que permita establecer la concentración de catalizador y el tiempo de operación de la reacción para minimizar los costos del proceso.

Shukla y Pushpavanam (1994) reportaron que, para concentraciones de catalizador de 1 y $5 \%$, el sistema reactivo es sensible. Esto lo hicieron empleando los criterios de Barkelew (1959) y de Villermaux y Georgakis (1991).Sin embargo, no definieron cuantitativamente los valores de las variables operacionales que limitan la sensibilidad paramétrica del sistema reactivo. En el presente trabajo se establece una concentración crítica de anhídrido acético en función de la concentración de catalizador. 


\section{CONCLUSIONES}

A partir de los resultados obtenidos, se pueden indicar las siguientes conclusiones: 1) Se lograron extender los criterios de Froment et al. (2011) para el modelo de un reactor batch, con temperatura de pared constante, en el que ocurre una única reacción irreversible de pseudo-primer orden. Estos criterios están basados en propiedades matemáticas del sistema reactivo y no utilizan valores arbitrarios. 2) El primer criterio se basa en determinar la trayectoria que corta al máximo de la curva de puntos calientes, mientras que el segundo se fundamenta en los puntos de inflexión de los perfiles de temperatura. Ambos constituyen herramientas útiles para establecer condiciones de operación segura. 3) Usando ambos criterios, se pueden hallar las concentraciones críticas de alimento de anhídrido acético. Estas concentraciones se pueden usar como punto de partida para determinar las condiciones que permitan realizar el proceso de forma segura y también, generar escenarios en los que se minimicen los gastos de operación. No obstante, esta clase de estudios requiere análisis posteriores más detallados.

\section{AGRADECIMIENTOS}

Este trabajo se ha desarrollado con el apoyo de la DIMA (Dirección de Investigaciones de la Universidad Nacional de Colombia, Sede Manizales) y del El Departamento Administrativo de Ciencias, Tecnología e Innovación (COLCIENCIAS, - Programa Jóvenes Investigadores 2014).

\section{REFERENCIAS}

Adrover, A., y otros tres autores, "Explosion limits and runaway criteria: A stretching-based approach", doi:10.1016/j.ces.2006.11.007, Chemical Engineering Science, 62(4), 1171-1183, (2007)

Asprey, S.P., B.W. Wojciechowski, M.N. Rice, y A. Dorcas, "Applications of Temperature Scanning in Kinetic Investigations: The Hydrolysis of Acetic Anhydride",doi:10.1016/0009-2509(96)00306-5, Chemical Engineering Science, 51(20), 4681-4692, (1996)

Barkelew, C. H., "Stability of Chemical Reactors", Chem. Eng. Progr. Symp. Ser., 25, 37-46 (1959)

Bosch, J., y otros tres autores, "A Comparative Analysis between Temperature and Pressure Measurements for Early Detection of Runaway Initiation", doi:10.1016/j.jp.2004.07.003, Journal of Loss Prevention in the Process Industries, 17(6), 389-395 (2004)

Casson, V., y otros tres autores, "Comparison of criteria for prediction of runaway reactions in the sulphuric acid catalyzed esterification of acetic anhydride and methanol",doi:10.1016/j.jp.2011.09.002, Journal of Loss Prevention in the Process Industries, 25, 209 - 217, (2012)

Froment, G. F., K. B. Bischoff, y J. De Wilde, "Chemical Reactor Analysis and Design", ISBN 978-0-47056541-4, Wiley, USA (2011)

Haldar, R. y D. P. Rao, "Experimental studies on limit cycle behavior of the sulphuric acid catalysed hydrolysis of acetic anhydride in a CSTR", Chemical Engineering Science, 46(4), 1197-1200 (1991)

Haldar, R. y D. P. Rao, "Experimental Studies on Parametric Sensitivity of a Batch Reactor", doi: 10.1002/ceat.270150107, Chem. Eng. Technol., 15(1), 34-38 (1992)

Jayakumar, N.S., y otros tres autores, "Experimental and theoretical investigation of parametric sensitivity and dynamics of a continuous stirred tank reactor for acid catalyzed hydrolysis of acetic anhydride", doi:10.1016/j.compchemeng.2010.09.005, Computers and Chemical Engineering, 35(7), 1295-1303 (2011)

Jayakumar, N.S., A. Thomas, M., y J.N. Sahu, "Experimental and modeling of a non-isothermal CSTR to find out parameter regions and conditions causing input multiplicity for acid catalyzed hydrolysis of acetic anhydride",doi:10.1016/j.chemolab.2014.04.017, Chemometrics and Intelligent Laboratory Systems, 135, 213-222 (2014)

Kirk-Othmer, "Encyclopedia of Chemical Technology",doi: 10.1002/0471238961, $5^{\text {th }}$ Edition, Wiley Blackwell; (2007)

Maestri, F., y R. Rota, "Thermally safe operation of liquid-liquid semibatch reactors. Part I: single kinetically controlled reactions with arbitrary reaction order", doi:10.1016/j.ces.2004.12.046, Chemical Engineering Science, 60(12), 3309-3322 (2005) 
Morbidelli, M., y A. Varma, "A generalized criterion for parametric sensitivity: application to thermal explosion Theory”, doi:10.1016/0009-2509(88)87129-X, Chemical Engineering Science, 43(1), 91 - 102 (1988)

Poling, B. E., J. M. Prausnitz, y J. P. O'Connell, The Properties of Gases and Liquids, 5a Edition, A.20-A.34, McGraw-Hill, Nueva York, EE.UU., (2000)

Rogers, P., J.S. Chen, M.J. Zidwick, "Organic Acid and Solvent Production Part I: Acetic, Lactic, Gluconic, Succinic and Polyhydroxyalkanoic Acids"doi: 10.1007/0-387-30741-9_19, Prokaryotes, 1, 511-755 (2006)

Shukla, P. y S. Pushpavanam, "Parametric Sensitivity. Runaway, and Safety in Batch Reactors: Experiments and Models",doi: 10.1021/ie00036a039, Ind. Eng. Chem. Res., 33(12), 3202-3208 (1994)

Strozzi, F. y J. M. Zaldivar, "A general method for assessing the thermal stability of batch chemical reactors by sensitivity calculation based on Lyapunov exponents", doi:10.1016/0009-2509(94)E0067-Z,Chemi. Engin. Science, 49(16), 2681-2688 (1994)

Transparency Market Research. "Acetic Acid Market Growth 2012 - 2018", Consultado en enero de 2016. http://www.transparencymarketresearch.com/pressrelease/acetic-acid-market.htm! (2015)

Varma, A., M. Morbidelli, y H. Wu, "Parametric Sensitivity in Chemical Systems", http://www.cambridge.org/, ISBN: 9780511721779 , Cambridge Series in Chemical Engineering, Cambridge University Press. (1999)

Villermaux, J. y C. Georgakis, “Current problems concerning batch reactors”, Int. Chem., 31, 434 (1991)

Westerterp, K. R., y E. J. Molga, "No more runaways in fine chemical reactors", doi: 10.1021/ie030725m, Industrial \& Engineering chemistry Research, 43(16), 4585-4594 (2004)

Westerterp, K. R., M. Lewak, y E. J. Molga, "Boundary Diagrams Safety Criterion for Liquid Phase Homogeneous Semibatch Reactors”,doi: 10.1021/ie500028u, Ind. Eng. Chem. Res., 53(14), 5778-5791 (2014) 
